\title{
The Impact of Cervicogenic Headache on Patients AtTending a PRivate Physiotherapy Practice In CAPE TOWN
}

\begin{abstract}
Headache (HA) disorders constitute a public health problem which impacts on individuals and on society. The functional limitations and participation restrictions caused by chronic HA's may be a more reliable indication of the severity of the disorder than the intensity and HA pattern itself Evaluation of all these parameters may assist holistic assessment, with impact

DIENER I , BSc'

1 Private Practice and part-fime Lecturer at the University of the Western Cope on the health care of chronic HA sufferers. A quantitative retrospective study of 450 patients' perceptions of the impact of chronic recurrent cervicogenic HA (CRCHA) was done by reviewing clinical notes made at first consultation. Bio-demographic data, Total Pain Pattern and impact of HA's on function, relationships and emotions were recorded using Likert scales. High scores were reported for severe functional disability (60\% of the subjects), for considerable emotional handicap (41\%) and for negative impact on close relationships (86\%). Decreased productivity was reported by $89 \%$ of the population studied. Subjects expressed emotions of anxiety (75\%), anger (48\%) and helplessness (62\%). CRCHA has a severe impact on the health-related quality of life of individuals. A combination of these results and existing instruments is currently being used to develop a measuring instrument reflecting a bio-psychosocial approach to assessment. This may improve physiotherapy management of CRCHA patients and may facilitate outcome-based research in this field.
\end{abstract}

\section{KEY WORDS: CERVICOGENIC HEADACHE, FUNCTIONAL LIMITATION, PARTICIPATION RESTRICTION, QUALITY OF LIFE ASSESSMENT}

\section{INTRODUCTION}

This study was done as part of a process of developing a holistic instrument to assess the severity and impact of chronic (longer than one year), recurrent (once a month or more) cervicogenic headache (As described by Edeling, 1994; Jull, 1997) on patients. When using a biopsychosocial approach to health care, the impairment as well as the impact on functional activities and participation in life situations must be addressed. The World Health Organisation (WHO) refers to the consequences of impairment (abnormality of body function) in terms of limitation of activities (previously described as functional disability) and restriction of participation (previously described as handicap) (WHO, 1999). Measuring headacherelated disability may provide physiotherapists with information that can help in determining which treatment options may be optimal for the patient. This process is challenging because of the wide spectrum of intensity and frequency of attacks, and variation in impact of the impairment on individuals. There is a dearth of information on the impact of headache (HA) on the quality of life of individuals suffering from migraine and tension-type headache (TTHA) (Blomkvist et al, 1997; Davies et al, 1999; Fishman and Black, 1999; Michel et al, 1999; Peterson-Braun and Gobel, 1994). However, no studies were found which included a group of individuals suffering from CRCHA.

The author, while assessing HA patients, experienced a need for an instrument specifically designed to measure the impact of CRCHA on the quality of life of patients. Physiotherapists see themselves, in a broad context, as trying to influence the ability of the patient to function, to cope with the disorder and to manage the associated distress. Although several instruments developed to measure disability resulting from low back pain, neck pain and chronic pain are currently used by physiotherapists, these do not include specific questions to measure the functional and psychosocial impact usually reported by individuals seeking treatment for CRCHA. Instruments to assess the quality of life and functional limitation in individuals with migraine (described as severe, episodic, short duration episodes of HA) have been developed and validated (Davies et al, 1999; Stewart et al, 1999; Michel et al, 1999). Instruments have also been developed for assessing and comparing quality of life in groups with migraine, cluster HA (described as short very severe episodic attacks of HA), and TTHA (described as longer-lasting moderate intensity HA) (Holroyd et al, 1997; Jacobson et al, 1994; Kryst and Scherl, 1994). The contents of these and of generic healthrelated quality of life questionnaires may facilitate the development of a measuring instrument for CRCHA. Although the International Headache Society (IHS)(1988) has classified

\section{CORRESPONDENCE TO:}

I Diener

16 Formosa Street, Stellenbosch 7600

Tel: (012) 887-1804

Fax: (012) 883-2108

E-mail: idiener@icon.co.za 
headaches into numerous categories, the impact of chronic recurrent $\mathrm{HA}$ of any type on function, social relations and emotional life is not dictated by the hypothesized origin/etiology of the HA, but by its intensity, temporal pattern, chronicity and response to therapy, as well as the psychological make-up of the individual (Fishman and Black, 1999). The severity of the HA impacts on the coping response in most patients. This was confirmed in a study where a direct relationship was established between increased HA intensity and frequency, and decreased quality of life, in individuals experiencing migraine and TTHA (Jacobson et al,1994). They also established that self-perceived disability resulting from HA was similar for these two groups of patients.

In the physiotherapy literature, headaches are mostly assessed in terms of pain intensity and temporal pattern (Edeling, 1994; Diener, 1995; Jull, 1997; Niere, 1998). Intensity, frequency, duration and analgesic intake are used as outcome measures for the effect of treatment. Niere (1998) also used subjective findings, including the McGill Pain Questionnaire, to predict physiotherapy treatment outcome in patients suffering from specific cervicogenic HA pain patterns.

A study was therefore initiated to develop an instrument to assess the impact of CRCHA in terms of functional limitations and socio-emotional restrictions. As a first step, the impact of HA on patients complaining of cervicogenic HA had to be compared to what was found in the literature for other types of HA. This current study was therefore designed to perform a retrospective review of data obtained from individuals with CRCHA seen in the author's practice, to assess the impact of this type of HA on their activities, social relationships and emotions.

\section{METHOD}

A quantitative retrospective review of the impact of $\mathrm{HA}$ on individuals complaining of CRCHA was done in February 2000. All available records (clinical notes on first physiotherapy consultation) of patients with CRCHA who had visited the author's clinic from $1 / 1 / 1995$ to $31 / 12 / 1999$ (a total of 450 patients) were reviewed, and the following information was recorded for each patient:

1. Bio-demographic data (age, sex, occupation/daily work)

2. Current Total Pain Pattern (according to Edeling, 1994)

- During the interview the patient was asked to rate the pain, the frequency of the headache episodes and the response to pain medication.

- Intensity of pain was scored on a Likert scale as $1=$ Mild, $2=$ More than mild, $3=$ Moderately severe, $4=$ Severe, and $5=$ Intolerable, suicidal.

- Frequency of episodes was scored as $1=$ Once per month, $2=2-3$ per month, 3 = Weekly, 4 = Daily, and $5=$ Continuous.

- Response to analgesics was scored as 1 = Mild analgesic abates pain, 2 = Mild analgesic lessens pain, 3 = Strong analgesic abates pain, $4=$ Strong analgesic lessens pain, and $5=$ No medication affects pain.

3. Self-perceived impact of the $H A$ on the function, social relationships and emotions.

- During the initial interview patients were asked how their function, relationships and emotions (in general) had been influenced by their HA. The responses were scored on Likert scales as $1=$ No impact/influence at all, 2 = Yes, but little impact, 3 = Yes, considerable impact, and $4=$ Yes, debilitating impact.

The specific subcategories for impact on function were days absent from work, loss of productivity in paid work, ability to do daily chores, restriction in taking part in sport and recreational activities, and ability to do future planning. The subcategories for relationships included their closest friend/spouse, dependants, friends and colleagues.

- Emotions recorded included feelings of depression, isolation, anxiety, helplessness, suicidal thoughts and anger. These were each scored with a yes or no response.

- Descriptive statistics were used to analyse the results.

\section{RESULTS}

\section{Bio-demographic data}

The records of 450 patients were reviewed: 352 females and 98 males. The average age was 38,9 years (range $=15-76$ ). The occupations of the patients included office personnel working with computers $(31 \%)$, housewives (22\%), musicians playing the violin, piano or flute $(16 \%)$, students $(16 \%)$, and individuals working with their hands in studios and factories (15\%).

\section{Current Total Pain Pattern (intensity, frequency, response to analgesics)}

Table 1 portrays the percentages of patients that complained of the different pain intensities, frequencies of episodes and responses to analgesics (See Method for explanation of scores).

\section{Table 1}

Seventy seven percent of the 450 patients experienced severe (4) to intolerable (5) pain. The mean score was $4.0(\mathrm{sd}=0,7)$. Similarly $47 \%$ of the patients experienced HA either on a daily basis (4) or continuously (5). The mean score on frequency of episodes was 3.3 ( $\mathrm{sd}=1 . \mathrm{I}$ ). Eighty percent of the patients reported that they needed strong analgesics to lessen (4) or abate (3) the pain (Mean score $=3.6$, $\mathrm{sd}=0.8$ ). Fourteen percent of patients found no relief with any medication.

TABLE 1. Group's average HA intensity, frequency of episodes and response to medication.

\begin{tabular}{|l|c|c|c|c|c|}
\hline & $\mathbf{1}$ & $\mathbf{2}$ & $\mathbf{3}$ & $\mathbf{4}$ & $\mathbf{5}$ \\
\hline Pain Intensity & $0 \%$ & $1 \%$ & $22 \%$ & $53 \%$ & $24 \%$ \\
\hline Frequency of episodes & $8 \%$ & $12 \%$ & $33 \%$ & $34 \%$ & $\cdot 13 \%$ \\
\hline Response to pain medication & $0 \%$ & $6 \%$ & $41 \%$ & $39 \%$ & $14 \%$ \\
\hline
\end{tabular}


TABLE 2. General impact of CRCHA on function, relationships and emotions

\begin{tabular}{|l|c|c|c|c|}
\hline & $\mathbf{1}$ & $\mathbf{2}$ & $\mathbf{3}$ & $\mathbf{4}$ \\
\hline Function & $0 \%$ & $8 \%$ & $32 \%$ & $60 \%$ \\
\hline Relationships & $0 \%$ & $11 \%$ & $54 \%$ & $35 \%$ \\
\hline Emotions & $3 \%$ & $24 \%$ & $41 \%$ & $32 \%$ \\
\hline
\end{tabular}

\section{Self-perceived impact of HA on function, social relationships and emotions.}

Table 2 portrays the percentages of patients that complained of the different scores of impact on their function, relationships and emotions (See Method for explanation of scores).

\section{Table 2}

Most $(92 \%)$ of the patients complained of a considerable (3) to disabling (4) effect on their function ( mean $=3.5$; $\mathrm{sd}=0.6$ ). A considerable negative (3) or debilitating (4) effect on their relationships was reported by $87 \%$ (mean $=3.2$; $\mathrm{sd}=0.6)$. A large percentage $(73 \%)$ of patients complained that their emotions were markedly (a score of 3 or 4 ) affected by their HA (mean $=3.0$; $\mathrm{sd}=0.8$ ).

\section{Specific functional limitations and socio- emotional restrictions.}

Graph 1 depicts the distribution of patients who perceived CRCHA to have an impact on specific functions, relationships and emotions.

\section{Groph 1}

From this graph it can be seen that the aspect of function perceived to be affected by the most patients was a loss of productivity ( $89 \%$ of subjects). All other aspects of function as recorded in the physiotherapy notes, were perceived to be affected by a smaller number of patients.

The relationship with a spouse or closest friend was perceived to be affected by more patients $(86 \%)$ than any of the other relationships. Relationships with colleagues were, however, also reported to be affected by a large number of patients.

Emotions of anxiety and feelings of helplessness were experienced by a large percentage of patients $(75 \%$ and $62 \%$ respectively). It is noteworthy that $12 \%$ (54 out of 450 patients) indicated that they experienced suicidal thought directly related to the CRCHA.

See Graph 1 for other results.

\section{GRAPH 1.Distribution of perceived impact of CRCHA on functions, relationships and emotions}

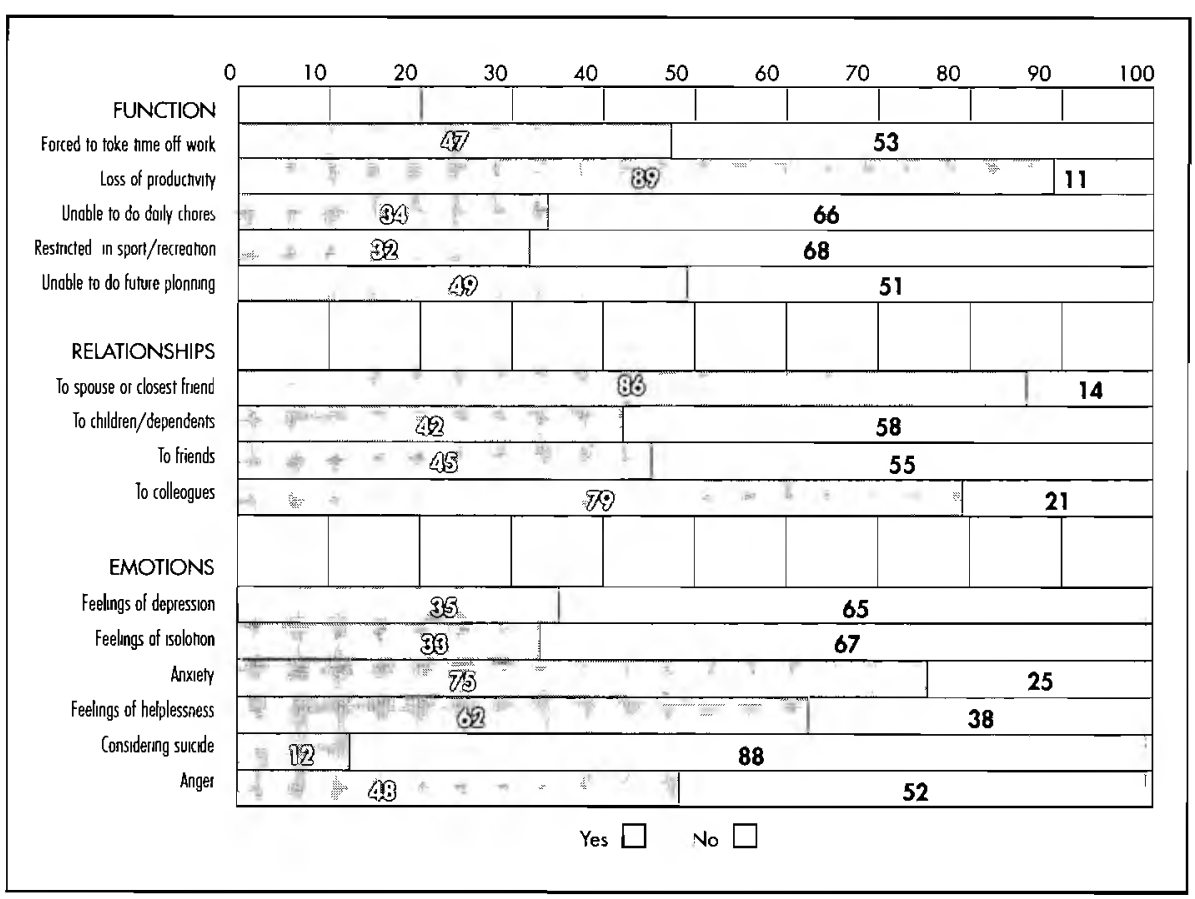

\section{DISCUSSION}

Although this study was done retrospectively, the results seem to agree with the literature on similar research into the impact of other types of HA on quality of life.

\section{Functional impact}

In a study of 177 (mostly) migraine sufferers by Stewart et al (1999), it was found that high pain intensity and frequency led to high functional disability, as portrayed in absenteeism and decreased productivity. Petersen-Braun and Gobel (1994) established that in $89 \%$ of 470 subjects migraine and TTHA interfered with work efficiency. The current study reports that in $89 \%$ of 450 patients productivity was influenced. Absenteeism was reported by $25 \%$ of their subjects, while the current study reports absenteeism in $47 \%$ of patients. Michel et al (1999) established that of 231 working migraineurs, $20 \%$ had at least one period of absenteeism per month. These figures are confirmed by other disability studies on individuals suffering from migraine and TTHA (Davis et al, 1999; Fishman and Black, 1999). The cost of providing healthcare for persons experiencing chronic $\mathrm{HA}$ is substantial (Fishman and Black, 1999), and the economic consequences of lost or reduced productivity in this group of patients may be greater than previously believed.

Functional disability may also emerge in day-to-day activities. In this study $34 \%$ reported interference with their daily chores, and $32 \%$ with their participation in sport/recreation. Regular cancellation of family and social activities as a result of chronic TTHA has also been reported by Davis et al (1999). Petersen-Braun and Gobel (1994) found that most subjects reported their severe migraines to be on week-ends. This interfered with normal leisure activities. In the current study $49 \%$ of patients reported interference with their ability to plan activities for the future. According to Blomkvist et al (1997) recurrent HA sufferers (cluster and migraine HA) often reported significantly poorer ability to anticipate future activities.

Absenteeism, productivity, ability to do household chores, restriction of 
leisure activities and future planning seem to be important consequences of CRCHA and therefore needs to be included in the assessment of these patients.

\section{Socio-emotional impact}

The impact of CRCHA on social and emotional aspects of patients' lives in the current study was similar to results reported in the literature. Interestingly, the closest personal relationships were affected in the largest percentage of patients, with work relationships following very closely. Relationships with children/dependants and with friends were also affected. The social and personal impact of HA was included in a national health survey done in Kentucky (Kryst and Scherl, 1994). The primary focus of the research was on social disability or the impact of severe migraine HA on interpersonal relationships with family, friends, co-workers and others. Their findings suggest that serious HA sufferers may be adapting to their illness by restricting activity in areas they perceive more flexible than work, such as family and social life. Since interpersonal relationships and social interaction often bring satisfaction to the individual and enhance quality of life, HA patients who forgo these activities may experience a decrease in social support, perhaps leading to increased stress and isolation, and an increase in headache. PetersenBraun and Gobel (1994) collected information from 470 migraine and 321 TTHA patients. Both types of HA were found to cause severe interference with their quality of life, their fitness for work, and leisure-time activities. Significantly reduced intra-family openness was found in families where the mother suffered from migraine (Blomkvist et al, 1997).

In this study several patients reported that they experienced feelings of depression $(35 \%)$, isolation $(33 \%)$, anxiety $(75 \%)$, helplessness $(62 \%)$, anger (42\%) and thoughts of suicide ( $12 \%$ ) as a result of their HA.

Depression as a self-perceived result of chronic migraine and TTHA was reported in several quality of life assessment studies (Jacobson et al, 1994; Kryst and Scherl E, 1994; Holroyd et al,
1997). The development of specific personality characteristics as a consequence of suffering from episodic $\mathrm{HA}$ is suggested by studies in the field (Blomkvist et al, 1997; Niere, 1998).

The societal burden of headaches may be enormous, but the significance of this disorder has long been overlooked, probably because of the episodic nature and the lack of mortality attributed to the condition. In patients with chronic HA disorders, physical, laboratory, and radiographic examinations are generally normal. For this reason clinicians often perceive the limitations associated with $\mathrm{HA}$ as less significant than those associated with other chronic conditions. Pain, in itself, is not a reliable measure for dysfunction nor for the outcome of management. The individual's perception of pain is greatly influenced by previous experience of pain, emotional factors and cultural beliefs. It was found that the physiotherapists' perception of patients' pain and other evaluative findings, are also influenced by personal and professional experience and individual psychological factors (Askew et al, 1998). If the outcome of physiotherapy (and likewise medication and other regimes of management) is measured by improvement in function, social participation and emotional state, in addition to changes in pain intensity and temporal pattern, it will make much more sense to therapist and patient. A reliable and valid measure of outcome of physiotherapy treatment may contribute to the profession's credibility and public image and may facilitate outcome research in physiotherapy.

\section{CONCLUSION}

It can be concluded from the results of this retrospective study, and from the literature reviewed, that individuals with CRCHA experience considerable restriction of daily function, limitation of social participation and emotional distress. If questions about all these effects are combined in a single questionnaire, it might be possible to measure the total impact of CRCHA on individuals. The addition of this to assessment of intensity and temporal pain pattern may be a more reliable indication of the severity of the disorder. Such an instrument is currently being developed to reflect this bio-psychosocial approach to assessment. This may facilitate outcome-based management of patients suffering from the disabling and sometimes debilitating impact of CRCHA on their quality of life.

\section{ACKNOWLEDEMENTS}

Prof. Dele Amosun, Department of Physiotherapy, University of the Western Cape, for his much appreciated advice.

\section{REFERENCES}

Askew R, Kibelstis C, Overbaugh S, Walker S, Nixon-Cave K, Shepard KF 1998 Physical therapists' perception of patients' pain and its effect on management. Physiotherapy Research Journal 3:37-57

Blomkvist V, Hannerz J, Orthgomer K, Theorell T 1997 Coping style and social support in women suffering from cluster headache or migraine. Psychotherapy and Psychosomatics 66:150-154

Davies GM, Santanello N, Gerth W, Lerner D, Block GA 1999 Validation of a migraine work and productivity loss questionnaire for use in migraine studies. Cephalalgia 19:497-502

Diener I 1995 Headache: Relief is possible. South African Family Practice 16:734-737

Edeling J 1994 Manual Therapy for Chronic Headache (2nd edit). Butterworth-Heinemann Ltd, Oxford England

Fishman P, Black L 1999 Indirect costs of migraine in a managed care population. Cephalalgia 19:50-57

Holroyd KA, O'Donnell F, Hill K, Lipchik G, Pinnell C, Malinoski P 1997 Assessing headache severity in treatment planning and outcome assessment. Headache 37:312 - 316 International Headache Society - Headache Classification Committee (1988) Classification and diagnostic criteria for headache disorders, cranial neuralgias and facial pain. Cephalalgia 8(suppl 7): 1-96.

Jacobson GP, Ramadan NM, Aggarwal S and Newman C 1994 The Henry Ford Hospital Headache Disability Inventory (HDI). In: Olesen, J (Ed): Headache classification and epidemiology, pp335-344. Raven Press, New York

Jull GA 1997 Management of cervical headache. Manual Therapy 2:182-190 
Kryst S, Scherl E 1994 A population-based survey of the social and personal impact of headache. Headache 34:344-35

Michel P, Dartigues JF, Duru G, Moreau, Salamon R, Henry P 1999 Incremental absenteeism due to headaches in migraine: Results from the Mig-Access French national cohort. Cephalalgia 19:503-510

Niere KR 1998 Can subjective characteristics of benign headache predict manipulative physiotherapy treatment outcome? Australian Journal of Physiotherapy 44:87-93

Petersen-Braun M, Gobel, H 1994 Degree of disability, affliction, loss of free time, and loss of working hours in patients with migraine and tension-type headache in Germany. In: Olesen $\mathbf{J}$ (edit) Headache classification and epidemiology. 4: 351-354. Raven Press. New York

Stewart WF, Lipton RB, Kolodner K,
Liberman J and Sawyer J 1999 Reliability of the migraine disability assessment score in a population-based sample of headache sufferers. Cephalalgia 19:107-14

World Health Organization 1999 International Classification of functioning and disability. ICIDH-2. Beta-2 Draft. Geneva

\section{South West London}

\section{Community NHS Trust}

has many of the professional benefits associated with an acute trust but within a community setting. We provide a variety of physiotherapy services across a large geographical areat on the outskirts of central London.

- 80 Physiotherapists

- Actively involved in Local \& National Initiatives

- Commitment to staff development

- Comprehensive IST \& Internal courses

- Funding for external courses

- Plentiful parking

- No weekend / On-call working

- Crèche

- Staff Gym at Queen Mary's site

- Hospital accommodation

\section{QUEEN MARY'S HOSPITAL,} ROEHAMPTON LANE

ROEHAMPTON, LONDON, SW15 5PN

This trust is firmly committed to equal opportunities, flexible working patterns, and a non-smoking environment.

\section{South West London Community NHS Trust \\ QUEEN MARY'S HOSPITAL PAEDIATRIC PHYSIOTHERAPY SERVICE}

\section{SENIOR I PHYSIOTHERAPIST}

Full time - 36 hours per week

(part-time/ job share considered)

$£ 23,444-£ 27,049$ pro rata

(inc. of Inner London Weighting \& Supplement)

This is an excellent opportunity for an experienced Paediatric Senior II physiotherapist looking for new challenges, or an existing Senior I keen to further develop their skills within the dynamic, forward thinking Paediatric Community Team based at Queen Mary's Hospital, Roehampton.

Your responsibilities will include:-

- Managing a team of physiotherapists at a school for children with special educational needs.

- Managing a varied caseload of children in mainstream schools, children's centres, nurseries and at home.

The post will provide you with the opportunity to further develop your clinical and managerial skills working alongside a team of experienced clinicians using a variety of approaches including Bobath, Conductive Education and Hare. The ability to work closely with children and their carers, education and health professionals as part of a team to develop continuing high standards of clinical care is essential.

There is a strong commitment to continuing professional development, clinical effectiveness and research.

Assistance with arranging a work permit is available.

For further information and/or an informal visit, please contact: Edwina Falls, Superintendent Paediatric Physiotherapist, Leon Gillis Children's Centre

Tel: 02083552443

e-mail: Edwina.Falls@swlct.sthames.nhs.uk

Closing Date: 28 February 2001 